\title{
ASFIXIA PERINATAL
}

\author{
Dolly Alexandra García MD*, Héctor Romero MD**, Alejandro Emilio Colmenares MD***
}

\section{Resumen}

Se revisa la literatura sobre asfixia perinatal debido a que no existe un consenso sobre su definición. La fisiopatológica actual no es operativa en la clínica, en donde se han barajado distintos criterios, como son: alteraciones de la frecuencia cardíaca fetal, presencia de líquido amniótico meconial, acidosis metabólica, bajas puntuaciones del Apgar y signos clínicos de lesión cerebral posasfíctica. Se analiza y se adopta la descrita por la Asociación Americana de Ginecología y Obstetricia (ACOG) y su par de pediatría (AAP). Así mismo se revisan las complicaciones posasfícticas en los recién nacidos y se discute la pertinencia del manejo, teniendo en cuenta la alta incidencia en países en vías de desarrollo y las nuevas terapéuticas que conducen a mitigar los daños que genera.

Palabras clave: asfixia perinatal, encefalopatía hipóxico isquémica, signos neurológicos, acidosis metabólica.

Abreviaturas: AP, asfixia perinatal; RN: recién nacido(s).

\section{PERINATAL ASPHYXIA}

\section{Abstract}

\begin{abstract}
A literature review on perinatal asphyxia was conducted for no consensus definition exists. The current definition based on its pathophysiology cannot be applied in clinical practice, where various criteria, such as: alterations in fetal heart rate, meconium in the amniotic fluid, metabolic acidosis, low Apgar scores and clinical signs of post asphyxiation cerebral lesion, have been described. The definition specified by the American College of Obstetrics and Gynecology (ACOG) and its pediatric peer association (AAP) was analyzed and adopted. Post asphyxiation complications in newborns were reviewed. Relevant management was discussed considering the high incidence of this condition in developing countries and the new therapies which lead to mitigate injuries caused by perinatal asphyxia
\end{abstract}

Key words: perinatal asphyxia, hypoxic ischemic encephalopathy, neurological signs, metabolic acidosis.

Fecha recibido: enero II de 2012 - Fecha aceptado: noviembre 15 de 2012

* Residente III de Pediatría. Hospital de San José. Fundación Universitaria de Ciencias de la Salud, Bogotá DC. Colombia.
** Pediatra. Instructor Asociado, Fundación Universitaria de Ciencias de la Salud, Bogotá DC. Colombia.

*** Epidemiólogo, pediatra Hospital de San José. Instructor Asistente, Fundación Universitaria de Ciencias de la Salud, Bogotá DC. Colombia. 


\section{Introducción}

A pesar del amplio uso del término asfixia perinatal no existe un consenso sobre su definición pues la fisiopatológica no se utiliza en la clínica por la diversidad de criterios como son alteraciones de la frecuencia cardíaca fetal, presencia de líquido amniótico meconial, acidosis metabólica, bajas puntuaciones del Apgar, signos clínicos de lesión cerebral posasfíctica y manifestaciones extraneurológicas como es el fracaso para iniciar movimientos respiratorios tras el nacimiento. ${ }^{1}$

Es de gran importancia en el ámbito médico tener un claro concepto sobre AP y definir el contexto clínico que esta implica, no solo como secuela neurológica de seguimiento sino también por la repercusión hemodinámica que implica a corto, mediano y largo plazo. Es por esto que se revisa la literatura para realizar una aproximación a la definición y demás contextos descritos antes. Se buscaron los términos definidos por descriptores en ciencias de la salud (DECS), se realizó la revisión en bases de datos de PUBMED, HINARI, OVID con términos en inglés y base de datos en español de LILACS y SCIELO, usando los conectores $A N D$ para cada una de las complicaciones de AP y OR para encefalopatía hipóxica isquémica. Se revisaron 86 referencias bibliográficas, 59 artículos con texto completo y 27 resúmenes; se incluyeron 59 artículos con base en la pertinencia y la novedad de cada uno de los manuscritos.

\section{Definición}

Desde el punto de vista fisiopatológico se puede definir como la agresión producida al feto o RN por la falta de oxígeno o la falla en la perfusión tisular adecuada, que suele asociarse con acidosis láctica e hipercapnia. ${ }^{1}$ El diagnóstico clínico de la AP se basa en varios criterios, los dos principales son evidencia de depresión cardiorrespiratoria y neurológica (definida como una puntuación de Apgar menor de 7 a los cinco minutos después del nacimiento) y compromiso hipóxico agudo con acidemia $(\mathrm{pH}$ arterial $<7$ o una base exceso $>12$ $\mathrm{mmol} / \mathrm{l}){ }^{2}$
Desde el punto de vista fisiológico según Volpe se puede definir la AP como la insuficiencia de oxígeno en el sistema circulatorio asociada con grados variables de hipercapnia y acidosis metabólica; desde el punto de vista clínico se caracteriza por los siguientes componentes: acidosis metabólica $\mathrm{pH}<7.00$, Apgar de 0-3 después de cinco minutos y signos en el período neonatal en los sistemas nervioso central, renal, pulmonar, cardiovascular, gastrointestinal, metabólico y hematológico. ${ }^{3}$ Este es el criterio utilizado por la Asociación Americana de Ginecología y Obstetricia (ACOG) y su par de pediatría (AAP).

\section{Epidemiología}

La estimación de la incidencia de AP varía en función de las definiciones utilizadas, la AP severa (causa de muerte o deterioro neurológico significativo) es alrededor de 1/1.000 nacidos vivos. En países en vías de desarrollo, la AP es más común. Los datos de estudios de hospitales sugieren una incidencia de 5-10/1.000 nacidos vivos. Sin embargo, es probable que haya subestimación de lo real debido a un subregistro. ${ }^{4}$ La incidencia de la mortalidad por AP es cerca de $1 \%$ a $1,5 \%$ en la mayoría de los centros y por lo general se relaciona con edad gestacional y peso al nacer. Se presenta en $9 \%$ de los niños menores de 36 semanas de gestación y en $0,5 \%$ de bebés de más de 36 semanas de gestación. ${ }^{5}$

\section{Factores de riesgo}

La atención deficiente durante el parto puede resultar en una consecuencia grave, desde un niño discapacitado hasta la muerte, pero su relación causal es difícil de probar. ${ }^{6}$ Además del contexto psicológico de duelo por parte de los padres, los gastos por negligencia relacionados con el parto son muy elevados. La experiencia ha demostrado que la morbilidad perinatal se puede reducir mediante la mejora de la atención obstétrica y el cuidado neonatal. ${ }^{?}$

Los factores de riesgo anteparto descritos son enfermedad tiroidea de la madre, infecciones virales 
prenatales, preeclampsia severa, sangrado de la madre, anomalías de la placenta y restricción del crecimiento intrauterino. ${ }^{8}$ Algunos de los factores de riesgo de AP intraparto identificados son parto prematuro, líquido amniótico meconiado, estimulación con oxitocina, parto de nalgas y complicaciones del cordón. ${ }^{9}$

\section{Asfixia y genética}

Se ha determinado la frecuencia de un polimorfismo del gen de supresión, GSTT1 y GSTM1, que se logró mediante reacción en cadena de la polimerasa para la genotipificación de 245 recién nacidos a término en Ucrania, sin encontrar diferencias alélicas significativas de homocigotos. ${ }^{10}$ El estudio muestra que la AP severa puede desarrollarse como consecuencia de una predisposición genética, sin embargo debería ampliarse el estudio de la genética molecular para determinar esta asociación.

\section{Asfixia y cordón umbilical}

El estado ácidobase del feto es un componente importante en el establecimiento de la relación entre los acontecimientos durante el parto y la condición neonatal. El análisis de los gases de la sangre de la arteria umbilical del cordón se cree que es la mejor representación del estado ácidobase del feto inmediatamente antes del parto. El análisis de gases sanguíneos es capaz de confirmar de manera objetiva o excluir la presencia de acidemia perjudicial. ${ }^{11}$

La demanda de oxígeno, incluyendo el déficit de base (BD) y las concentraciones de lactato del feto, deben ser una medida directa del metabolismo anaeróbico. En la práctica muestran un lugar amplio e impreciso en relación con la encefalopatía neonatal. Por ejemplo, acidosis profunda $\mathrm{BD}>18 \mathrm{mmol} / \mathrm{l}$ a los treinta minutos de vida se asoció con encefalopatía moderada a severa en un $80 \%$ de pacientes, mientras en 21 casos no se produjo con BD <10-12 mmol/l. ${ }^{12}$ Sin embargo, los resultados son muy variables. Low y col. encontraron que menos de la mitad de bebés que nacen con una $\mathrm{BD}>16 \mathrm{mmol} / \mathrm{l}(\mathrm{y} \mathrm{pH}<7,0)$ desarrollan importante encefalopatía. $^{13}$
Por otra parte, un estudio que incluyó 17.978 recién nacidos encontró que el rango estadístico normal del $\mathrm{pH}$ sanguíneo de la arteria umbilical y la BE para los recién nacidos fue 7,20 $\pm 0,20$ y $-7,64 \pm 10,02$, respectivamente. El umbral patológico de $\mathrm{pH}$ o de la $\mathrm{BE}$ de asfixia neonatal es un rango en lugar de un punto fijo. El rango de distribución del pH sanguíneo de la arteria umbilical fue corregido en la clínica mediante la puntuación del Apgar, obteniendo los valores de asfixia neonatal de $<7.00,<7.20$ y $-8,-18$, en forma respectiva. $^{14}$

\section{Otros biomarcadores}

Durante y después de la exposición a hipoxia-isquemia, una variedad de marcadores bioquímicos se elevan en los fluidos corporales y son fáciles de acceder y medir. ${ }^{15}$ Dado que muchos se activan en el cerebro por la hipoxia, son útiles como marcadores centinela para la encefalopatía hipóxico isquémica el S100B, la enolasa neuronal específica (NSE), activita A, adreno medulina y la interleucina (IL)- $1 \beta$ e IL- $6 .{ }^{16}$

Todos estos biomarcadores son inducidos después de la hipoxia. Sin embargo, es importante tener en cuenta que pueden elevarse en otras situaciones. Los niveles de S100B, por ejemplo, aumentan en forma notable ante la restricción del crecimiento intrauterino o hipoxia crónica ${ }^{16}$, infección perinatal o inflamación, hipoperfusión central y periférica, parto traumático, lesiones neurales preexistentes, tratamiento con glucocorticoides prenatales, anestésicos y la prematurez. Además el cerebro no parece ser la única fuente de S100B y NSE, ya que puede liberarse en una gran variedad de tejidos, incluyendo el cordón umbilical y la placenta ${ }^{17}$, lo que indica que la investigación frente a bio-marcadores con alta sensibilidad y especificidad aún está en proceso.

\section{Asfixia perinatal y estrés oxidativo}

Ya que el estrés oxidativo desempeña un papel importante en el mecanismo patogénico del daño a los tejidos en la hipoxia isquémica, los marcadores se 
están convirtiendo en una herramienta de pronóstico potencial. Son varios los que están bajo investigación, de los cuales la malondialdehído (MDA), que es un producto de la peroxidación de los lípidos, y por otro lado las proteínas de tipo carbonilos, reflejan en general el estrés oxidativo.

La MDA y los valores de proteínas carbonilo se han encontrado mayores en aquellos pacientes con resultados adversos del desarrollo neurológico que en normales, sin valor estadístico significativo pero importantes en la clínica. Su medición puede utilizarse como predictora de los resultados inmediatos en la $\mathrm{AP}$, aunque su papel en el desarrollo futuro aún no se ha determinado. ${ }^{18}$

\section{Asfixia y falla multiorgánica}

Al estudiar los desenlaces de 72 historias consecutivas de RN con diagnóstico de AP, Ancel y col, encontraron que el compromiso de uno o más órganos se produjo en el $82 \%$ de los niños y el sistema nervioso central fue el más implicado (72\%). La afectación renal se produjo en $42 \%$, pulmonar en $26 \%$, cardíaca en $29 \%$ y gastrointestinales en $29 \%$. El $15 \%$ tenía insuficiencia renal y el $19 \%$ insuficiencia respiratoria. Las puntuaciones de Apgar a uno y cinco minutos fueron los únicos factores perinatales relacionados con el número y la gravedad de órganos afectados, el valorado a los cinco minutos tuvo la mayor asociación independiente. Llama la atención que en el estudio no se halló ninguna relación o disfunción orgánica con el pH sanguíneo arterial del cordón umbilical, líquido amniótico, anomalías del cordón umbilical y la presentación o el tipo de parto. ${ }^{19}$

El Colegio Americano de Obstetras y Ginecólogos y la Sociedad de Obstetras y Ginecólogos de Canadá, definieron unos criterios para determinar la presencia de encefalopatía hipóxica isquémica posparto. ${ }^{20}$

1. Uno o más de los siguientes: a) a los 5 minutos $A p$ gar $<5$, b) acidosis metabólica (análisis de gases en la primera hora después del nacimiento) con base exceso $>16 \mathrm{mmol} / \mathrm{l}$ y c) retraso en el inicio de la respiración durante cinco o más minutos.
2. Necesidad de ventilación mecánica en el nacimiento.

3. Evidencia de encefalopatía incluyendo alteración del estado de la conciencia y/o convulsiones.

4. Disfunción de otros órganos.

Asfixia y encefalopatía hipóxico isquémica: la hipoxia isquémica es la causa más común de enfermedad neurológica durante el período neonatal. Se asocia con alta mortalidad y morbilidad, incluyendo parálisis cerebral, discapacidad intelectual y convulsiones. ${ }^{5}$ Es una enfermedad multiorgánica e incluye circunstancias que afectan el flujo sanguíneo cerebral en el feto y el recién nacido que ponen en peligro el suministro de oxígeno al cerebro. La evaluación y gestión de estas complicaciones es una parte integral del tratamiento de la AP. ${ }^{3}$

Pasos de una lesión cerebral poshipóxica: bajo condiciones anaeróbicas el gasto metabólico de la producción de ATP es alto, dando lugar a una ruptura del equilibrio de energía que agota a las células del cerebro, necesaria para procesos metabólicos en neuronas y células gliales.

La necrosis es sólo uno de los mecanismos de muerte celular, la hipoxia o la isquemia cerebral la generan y también parece que contribuyen a la muerte celular que ocurre días o semanas después de la lesión. ${ }^{21}$

Hay una serie de posibles mecanismos en la generación de radicales libres bajo condición hipóxica. Durante esta el aumento de calcio intracelular como resultado de la activación excesiva de NMDA y no de sus receptores, es crucial en la hipoxia inducida por excitotoxicidad. La elevación del calcio inicia una serie de eventos bioquímicos que podrían conducir a la generación de radicales y muerte celular. ${ }^{22}$

Después de la isquemia, el óxido nítrico neuronal tiene efectos tóxicos, sin embargo el del endotelio presenta acción protectora en el cerebro. La lesión cerebral hipóxica se asocia con la formación de este óxido y estudios recientes sugieren que puede reaccionar con el anión superóxido para formar peroxinitrito y de esta forma causar la neurotoxicidad. ${ }^{23}$ 
Otro papel importante es el que juegan las caspasas que son una familia de proteasas cisteinilaspartato responsables de la iniciación y ejecución de la apoptosis. Se dividen en dos clases principales: la tipo I con caspasas 8,9 y 10 , las cuales se activan en la fase temprana de la apoptosis y las II tales como la 3, 6 y 7 responsables de la apoptosis celular, denominadas también caspasas efectoras. $^{24}$

\section{Nivel electrolitico}

El cuerpo debe mantener un nivel óptimo de los electrolitos en la sangre. Los recién nacidos asfixiados tienen una diferencia estadística significativa de los niveles de potasio y calcio en suero. Sin embargo, no se menciona si la reducción de estos dos es proporcional a la gravedad de la asfixia o si ello es debido al azar. ${ }^{25}$ Los RN que cursan con AP desarrollan hiponatremia (concentración sérica menor de $130 \mathrm{mEq} / \mathrm{l}$ ) e hipocalcemia (nivel inferior a $7 \mathrm{mg} / \mathrm{dl}$ ) poco después del nacimiento. Hasta el momento el nivel de potasio no está definido con claridad y se requieren más estudios para encontrar algún punto de corte que requiera atención de emergencia. ${ }^{26}$

La hipocalcemia es una de las complicaciones electrolíticas más comunes y frecuentes en el RN. El calcio iónico es fundamental para muchos procesos bioquímicos incluyendo coagulación, excitabilidad neuromuscular, integridad de la membrana celular y muchas de las actividades enzimáticas celulares. ${ }^{27} \mathrm{La}$ de aparición temprana, que se presenta en las primeras 72 horas, requiere tratamiento con suplementos de calcio durante al menos 72 horas. Por el contrario, la de inicio tardío que ocurre por lo regular después de siete días, exige terapia a largo plazo. ${ }^{27}$

\section{Nivel cardíaco}

La lesión celular miocárdica en los $\mathrm{RN}$ puede ocultarse a la clínica. En los últimos años se ha mostrado un creciente interés en el uso de la troponina I cardíaca (cTnI) en AP y lesión miocárdica hipóxica. Altos niveles podrían utilizarse como marcadores de la asfixia e incluso como predictores de los resultados futuros o de la mortalidad. ${ }^{28}$
Por otro lado, son importantes los niveles de la $\mathrm{N}$-terminal del péptido natriurético cerebral lpro(NT-proBNP) y del glucógeno fosforilasa isoenzima $\mathrm{BB}$ (GPBB) en los RN con asfixia complicada, por la lesión miocárdica. Tanto NT-proBNP y GPBB se pueden utilizar como biomarcadores de lesión miocárdica en los RN con asfixia. La medición de los niveles plasmáticos es útil en la identificación precoz de daño miocárdico y en la evaluación de la gravedad en los RN con asfixia. ${ }^{29}$

\section{Cambios ecocardiográficos}

Rajakumar y col. demostraron una incidencia de insuficiencia tricuspídea de $23,3 \%$. Puede deberse a isquemia del músculo papilar o hipertensión pulmonar persistente. ${ }^{30}$ Sin embargo, a lo largo de la revisión se han encontrado incidencias menores como la de Rowe de $12 \% .{ }^{31}$ La amplia variación puede explicarse por el tipo de los casos seleccionados (más severos) y la presencia o ausencia de hipertensión pulmonar. ${ }^{30} \mathrm{La}$ disfunción ventricular derecha debida a isquemia se presenta en $30 \%$ de pacientes con AP. La hipocinesia derecha es más frecuente que la izquierda, debido a la mayor masa muscular y a la elevación de la presión pulmonar en el período neonatal temprano. ${ }^{30}$

\section{Nivel respiratorio}

La hipertensión pulmonar persistente del RN puede ocurrir con AP, ya sea por efectos directos de la hipoxia/isquemia sobre la función pulmonar arterial o en forma indirecta debido a que ambas están asociadas con el síndrome de aspiración de meconio o sepsis perinatal. Lapointe y col. en una revisión de la literatura concluyen que la hiperventilación se debe evitar, la terapia con bicarbonato no está probada, y que tanto la hipoxia y la hiperoxia deben ser evitadas. El óxido nítrico aumenta la presión arterial pulmonar y la perfusión sistémica. Los efectos de los agentes inotrópicos sobre la perfusión cerebral o los resultados son inciertos. ${ }^{32}$

\section{Nivel circulatorio}

La asfixia neonatal se asocia con disfunción de múltiples órganos debido a una hipoxia isquémica posterior. 
El sistema cardiovascular se afecta con frecuencia causando signos de shock, lo cual complica la transición circulatoria neonatal. La terapia con hipotermia puede mejorar el resultado de asfixia neonatal sin alteración negativa de la estabilidad cardiovascular. La terapia dirigida al sistema cardiovascular puede mejorar a corto plazo las medidas del flujo sanguíneo sistémico, pero hasta la fecha no se ha demostrado que lo haga a largo plazo..$^{33}$

\section{Nivel hepático}

Se ha encontrado daño hepático como lo reportó Tarcan y col. en un estudio retrospectivo en $56 \mathrm{RN}$ con AP, en donde se evidenció lesión del hepatocito basándose en los elevados niveles séricos de alanina amino transferasa (>100 U/l, dos veces superior), con normalización posterior. Veintidós de los pacientes tenían alguna lesión de hepatocitos. El sufrimiento fetal, la trombocitopenia, las convulsiones, los hallazgos patológicos en las imágenes del sistema nervioso central y una alta tasa de retraso del crecimiento intrauterino fueron los factores más significativos asociados con alteraciones del hepatocito. Este daño se correlacionó con mortalidad elevada. ${ }^{34}$ También se ha identificado relación de Apgar bajo y colestasis neonatal, que oscila alrededor del $7 \% .{ }^{35}$

\section{Nivel renal}

La insuficiencia renal aguda es una consecuencia común de la AP que ocurre hasta en $56 \%$. Una dificultad importante en el diagnóstico de esta enfermedad es la falta de una definición consensuada en el neonato debido con frecuencia a la escasez de determinadas variables medibles y los marcadores bioquímicos. ${ }^{36}$

En un estudio prospectivo se determinó la incidencia de insuficiencia renal en los neonatos asfixiados y la correlación de la severidad y el tipo de presentación con Apgar bajo. Noventa y ocho RN se reclutaron, setenta asfixiados y 28 controles sanos. La función renal se evaluó mediante la producción de orina, la microscopía de esta, los parámetros bioquímicos y los hallazgos ecográficos. Los bebés con insufi- ciencia renal se siguieron hasta cumplir seis meses de edad para detectar cualquier deterioro residual. El nitrógeno ureico y la creatinina sérica fueron mayores en los RN asfixiados en comparación con el grupo control. ${ }^{37}$

Los niveles de sodio sérico y su excreción fraccionada mostraron valores bastante diferentes en los RN asfixiados en comparación con los controles. De los 70 asfixiados, $33(47,1 \%)$ tenían insuficiencia renal que fue de tipo no oligúrica en $78 \%$ y oligúrica en $22 \%$. Se observaron alteraciones ecográficas con mayor frecuencia en aquellos con falla renal oligúrica y se asoció con mal pronóstico. Los parámetros renales se normalizaron en todos los $\mathrm{RN}$ a los seis meses de edad. La mortalidad fue mayor en insuficiencia renal oligúrica.

La insuficiencia renal es un problema importante en los RN asfixiados, la mayoría cursa con no oligúrica y la gravedad de la alteración renal y su función se correlacionan bien con el grado de asfixia. La oliguria, hiponatremia y exploración ecográfica anormal son malos signos pronósticos cuando es secundaria a asfixia al nacer. ${ }^{37}$ En la mayoría de los estudios se ha definido como creatinina sérica $>1.5 \mathrm{mg} / \mathrm{dl}$; sin embargo, no existe una definición universal aceptada. ${ }^{38}$ En 2007 una colaboración de investigadores de todas las principales sociedades de cuidado crítico y nefrología, propusieron el término de injuria renal aguda y un sistema de clasificación que comprende etapas como se evidencia en la Tabla 1. ${ }^{39}$

\begin{tabular}{|c|c|c|}
\hline \multicolumn{2}{|c|}{$\begin{array}{r}\text { Tabla 1. Estadios de la falla renal aguda } \\
\text { (traducido del original) }\end{array}$} \\
\hline Estadio & Creatinina en suero & Gasto urinario \\
\hline I & $>150-200 \%$ de la basal & $<0.5 \mathrm{ml} / \mathrm{k} / \mathrm{h} \times 6$ horas \\
\hline II & $>200-300 \%$ de la basal & $<0.5 \mathrm{ml} / \mathrm{k} / \mathrm{h} \times 12$ horas \\
\hline III & $>300 \%$ de la basal & $\begin{array}{r}<0.3 \mathrm{ml} / \mathrm{k} / \mathrm{h}>24 \text { horas o anuria } \\
>12 \text { horas }\end{array}$ \\
\hline
\end{tabular}




\section{Asfixia y síndrome hemolítico urémico}

El síndrome urémico hemolítico se define como la tríada de insuficiencia renal aguda, anemia hemolítica microangiopática y trombocitopenia; se supone que es una consecuencia del consumo de plaquetas en los sitios de lesión endotelial. ${ }^{40}$ Recordemos que los RN que cursan con AP e insuficiencia renal después del nacimiento debido a la necrosis tubular aguda o la necrosis cortical difusa, pueden asociarse con coagulación intravascular diseminada y consumo de plaquetas. Hasta la fecha no existe alguna investigación biológica que permita distinguir con claridad entre el síndrome y la coagulación mencionados. ${ }^{41}$ Así pues, la AP con insuficiencia renal puede presentar las características biológicas del síndrome hemolítico urémico. La coagulación intravascular diseminada puede asociarse con AP ya que el daño en las células endoteliales puede estar presente en dicho evento. ${ }^{42}$ Sin embargo, se necesitan más estudios para analizar la fisiopatología, los antecedentes y la necesidad de tratamientos específicos, en cuanto a la relación del síndrome y la presencia de AP.

\section{Asfixia e hipoglicemia}

El daño hipóxico isquémico cerebral es la consecuencia más importante de la AP. Afecta el metabolismo oxidativo neurológico que conduce a aumento del lactato, caída en el pH y disminución de compuestos de fosfatos (fosfocreatina, luego de adenosintrifosfato [ATP]), debido a la glucólisis anaeróbica para generar ATP. En condiciones anaeróbicas, una molécula de glucosa produce sólo dos de ATP, en comparación con 38 moléculas en condiciones aerobias. La glucosa se metaboliza muy rápido en anaerobiosis durante la asfixia para reducir al mínimo el agotamiento de la energía celular en todos los tejidos, incluyendo el cerebro. ${ }^{43}$ La hipoglucemia inicial es un importante factor de riesgo de lesión cerebral perinatal. La hiperuricemia (el nivel normal de ácido úrico en el $\mathrm{RN}$ va de 2 a 6,2 $\mathrm{mg} / \mathrm{dl}$ ) y muestra en forma indirecta la gravedad de las lesiones de los tejidos. ${ }^{44}$

\section{Asfixia y necrosis grasa subcutánea}

La necrosis subcutánea grasa del RN es una inflamación del tejido adiposo subcutáneo autolimitada que se desarrolla en la primera semana de vida del RN a término. ${ }^{45}$ Los factores de riesgo incluyen diabetes gestacional, preeclampsia, consumo materno de cocaína, asfixia al nacer, aspiración de meconio e hipotermia neonatal. ${ }^{46} \mathrm{La}$ desviación de la sangre lejos del tejido subcutáneo crea un ambiente de hipoxia y de hipotermia, que se cree que conduce a un ciclo de inflamación granulomatosa y necrosis del tejido adiposo, cuya composición presenta alta concentración de grasas saturadas. Los ácidos grasos tienen un punto de fusión superior $\left(64^{\circ} \mathrm{C}\right)$, haciéndolos más propensos a cristalizar en estrés por frío. ${ }^{46}$

\section{Evidencia en el tratamiento de asfixia perinatal}

\section{Alopurinol y bloqueadores del calcio}

Existen revisiones sistemáticas que no proporcionan pruebas suficientes acerca de los efectos de los antioxidantes en $\mathrm{RN}$ con AP, pese a su efecto reductor en la formación de radicales libres, sin que haya diferencias significativas en la mortalidad entre los niños tratados con alopurinol y placebo. ${ }^{47}$ Así mismo no hay evidencia que soporte el uso de bloqueadores de los canales de calcio en RN con AP, por el contrario se ha asociado con hipotensión clínica importante. ${ }^{48}$

\section{Corticoides}

Aunque los corticosteroides pueden reducir el edema cerebral en niños mayores o adultos con hipoxia cerebral, existen estudios que sugieren que no mejoran el pronóstico neurológico ni hay evidencia de algún efecto sobre la presión de perfusión cerebral en neonatos con AP.?

\section{Restricción hídrica}

Las principales recomendaciones actuales para restringir líquidos endovenosos se basan en datos procedentes 
del tratamiento de adultos y niños mayores o de modelos animales con hipoxia cerebral. ${ }^{49}$ La razón es que la restricción hídrica puede limitar el edema cerebral y ser importante en la patogénesis del daño neurológico después de la AP. Sin embargo, existe la preocupación de que la restricción excesiva puede causar deshidratación e hipotensión, lo que resulta en una disminución de la perfusión cerebral y daño tisular. ${ }^{2}$

\section{Inotrópicos y sulfato de magnesio}

No existen ensayos clínicos controlados aleatorios que reporten algún beneficio del soporte con dopamina u otros agentes inotrópicos en pacientes que presentan AP. ${ }^{50}$ Tampoco hay evidencia que sustente el uso de sulfato de magnesio ni hay diferencias significativas en el pronóstico neurológico a largo plazo con su uso o con agentes inotrópicos. ${ }^{51}$

\section{Reanimación con aire vs. oxígeno al $100 \%$}

Existen dos metaanálisis de varios ensayos clínicos controlados aleatorios que comparan la reanimación neonatal con aire vs. oxígeno al $100 \%$, en donde se evidenció una mayor supervivencia cuando la reanimación se comienza con aire. ${ }^{52}$ No hay estudios de largo plazo que comparan los resultados con diferentes concentraciones de oxígeno que no sea $100 \%$ o con aire ambiente. Se recomienda iniciar la reanimación en $\mathrm{RN}$ a término o prematuros logrando un valor de saturación de oxígeno en el rango intercuartil de la saturación preductal (clase IIb, LOE B). Estos objetivos pueden lograrse mediante aire o una mezcla con oxígeno utilizando la oximetría de pulso (clase IIb, LOE C). ${ }^{53}$ Cuando no hay disponibilidad de oxígeno mezclado, la reanimación debe iniciarse con aire IIb (clase, LOE B). Si el RN está con bradicardia (FC 60 por minuto) después de 90 segundos de reanimación con una concentración baja de oxígeno, se debe aumentar a $100 \%$ hasta lograr un ritmo cardíaco normal (clase IIb, nivel de evidencia B). ${ }^{53}$

\section{Pronóstico}

La AP en todo el mundo es una causa importante de muerte y de daño cerebral adquirido en el RN. El pronóstico depende de la gravedad de la asfixia. Sin embargo, hay escasez de datos basados en los resultados a largo plazo como parálisis cerebral, retraso del desarrollo, discapacidad visual y auditiva, así como problemas de aprendizaje y de comportamiento. La muerte temprana de la célula puede ocurrir en cuestión de minutos. La reanimación inmediata para restablecer el oxígeno y la circulación tiene como objetivo limitar la extensión de este daño. El tratamiento durante la fase posterior a la reanimación tiene como objetivo bloquear estos procesos, lo que limita el daño celular secundario y reduce al mínimo su magnitud..$^{54}$

El seguimiento del paciente con antecedente de AP en nuestro medio es complicado. Sin embargo se ha logrado en pacientes con Apgar menor de 7 a los cinco minutos, como el reportado por Stuart y col. que incluye 877.618 pacientes ${ }^{55}$, en donde se evidenció que uno de cada 44 con Apgar menor de 7 a los cinco minutos después del nacimiento, tiene más probabilidad de presentar deterioro cognitivo o bien requieren otras necesidades educativas especiales.

El estudio incluye además registros escolares de adolescentes con Apgar menor de 7 a los cinco minutos evidenciando mayor dificultad de graduación y aún más con honores, pero los datos fueron obtenidos del registro de nacidos vivos, por tanto la veracidad diagnóstica es dudosa. ${ }^{55}$

\section{Asfixia e hipotermia}

La posibilidad de que el enfriamiento terapéutico del cerebro del $\mathrm{RN}$ con encefalopatía posasfíctica puede limitar la muerte neuronal, ha sido considerado desde hace más de cuarenta años. ${ }^{56}$ Los estudios experimentales en modelos animales han demostrado que una disminución de $3^{\circ} \mathrm{C}$ en la temperatura después de una injuria hipóxica isquémica reduce la tasa metabólica neuronal y el nivel de energía celular secundaria. La hipotermia es una intervención neuroprotectora en el tratamiento de encefalopatía hipóxico isquémica tras la AP. Un metaanálisis de trece ensayos clínicos publicados evidencia que se asocia con una reducción de la mortalidad o de discapacidad del desarrollo neurológico en la infancia. ${ }^{57} \mathrm{En}$ la actualidad se investiga el 
efecto de enfriamiento de la cabeza directa (hipotermia sistémica moderada) o del cuerpo entero. Sin embargo, esta última no se recomienda fuera del contexto de ensayos clínicos controlados. ${ }^{2}$

\section{Asfixia y la medicina legal}

Demontis y col. con base en la experiencia italiana afirman que las demandas por pacientes con parálisis cerebral puede ser elevada y por ende se convierte en una tarea difícil para el obstetra y el neonatólogo defender la posible "negligencia" causante de terribles daños neurológicos. ${ }^{58}$ No es legal concluir que la acidosis grave se debe a una mala práctica médica ni aseverar que eventos agudos durante el parto conducen a hipoxia. Locatelli y col. informaron que la patología placentaria vascular es más común en pacientes con Apgar bajo a los cinco minutos y acidosis, que en aquellos con puntuaciones bajas sin acidosis. ${ }^{59}$ Por ello, los autores hacen énfasis en identificar cuando la causa de acidemia y Apgar bajo con su consecuente complicación neurológica no se debe a mala práctica médica sino a una posible alteración propia placentaria, así que el examen de este órgano podría arrojar datos valiosos en pro del cuestionamiento médico legal.

\section{Conclusiones}

La AP es un evento que por fortuna cursa con una baja incidencia, pero los efectos neurológicos y en general la falla multiorgánica son devastadores en aquellos RN que la presentan. Llama la atención que la incidencia en países en vías de desarrollo es casi diez veces más que en los desarrollados y que esta podría elevarse si la problemática del subregistro disminuyera, lo cual genera hipótesis que podrían dar solución a un evento de salubridad propiamente dicho.

Existe una diversidad de definiciones de AP, la más apropiada según criterios clínicos y fisiopatológicos es aquella adoptada por la Asociación Americana de Ginecología y Obstetricia (ACOG) y su par de pediatría (AAP). Por ello, todas las instituciones de salud deberían adherirse a dicho concepto para así disminuir y mitigar las consecuencias resultantes de un evento tan catastrófico. Todas aquellas acciones encaminadas a mitigar el daño secundario a AP presentan controversia, pero es claro que medidas preventivas como evitar los partos con $\mathrm{RN}$ postérmino independiente de la vía, así como una adecuada reanimación neonatal podrían disminuir la incidencia.

\section{Referencias}

1 González de DJ. [Definition of perinatal asphyxia in medical literature: the need of a consensus]. Rev Neurol. 2002 Oct 1;35(7):628-34

2 McGuire W. Perinatal asphyxia. Clin Evid (Online). 2007 Nov 7;2007

3 Hill A, Volpe JJ. Perinatal asphyxia: clinical aspects. Clin Perinatol. 1989 Jun;16(2):435-57.

4 Levene ML, Kornberg J, Williams TH. The incidence and severity of post-asphyxial encephalopathy in full-term infants. Early Hum Dev. 1985 May; 1 1(1):216.

5 Delivoria-Papadopoulos M, Marro PJ. Biochemical basis of hypoxic-Ischemic encephalopathy. NeoReviews. 2010 Apr $1 ; 1$ 1(4):el84-el93.

6 Jonsson M, Norden SL, Hanson U. Analysis of malpractice claims with a focus on oxytocin use in labour. Acta Obstet Gynecol Scand. 2007:86(3):315-9.

7 Berglund S, Grunewald C, Pettersson H, Cnattingius S. Risk factors for asphyxia associated with substandard care during labor. Acta Obstet Gynecol Scand. 2010;89(1):39-48.

8 Badawi N, Kurinczuk JJ, Keogh JM, Alessandri LM, O'Sullivan F, Burton PR, et al. Antepartum risk factors for newborn encephalopathy: the Western Australian case-control study. BMJ. 1998 Dec 5;317(7172):1549-53.

9 Milsom I, Ladfors L, Thiringer K, Niklasson A, Odeback A, Thomberg E. Influence of maternal, obstetric and fetal risk factors on the prevalence of birth asphyxia at term in a Swedish urban population. Acta Obstet Gynecol Scand. 2002 Oct;81(10):909-17.

10 Gorovenko NG, Rossokha ZI, Podolskaya SV, Pokhylko VI, Lundberg GA. The role of genetic determinant in the development of severe perinatal asphyxia. Tsitol Genet. 2010 Sep;44(5):41-6.

11 Blickstein I, Green T. Umbilical cord blood gases. Clin Perinatol. 2007 Sep;34(3):451-9

12 Wayenberg JL. Threshold of metabolic acidosis associated with neonatal encephalopathy in the term newborn. J Matern Fetal Neonatal Med. 2005 Dec:18(6):381-5.

13 Low JA, Lindsay BG, Derrick EJ. Threshold of metabolic acidosis associated with newborn complications. Am J Obstet Gynecol. 1997 Dec;177(6):1391-4.

14 [Multicenter clinical study on umbilical cord arterial blood gas param eters for diagnosis of neonatal asphyxia]. Zhonghua Er Ke Za Zhi. 2010 Sep;48(9):668-73

15 Gazzolo D, Abella R, Marinoni E, di IR, Li VG, Galvano F, et al. New markers of neonatal neurology. J Matern Fetal Neonatal Med. 2009:22 Suppl 3:57-61.

16 Ramaswamy V, Horton J, Vandermeer B, Buscemi N, Miller S, Yager J. Systematic review of biomarkers of brain injury in term neonatal encephalopathy. Pediatr Neurol 2009 Mar;40(3):21 5-26.

17 Routsi C, Stamataki E, Nanas S, Psachoulia C, Stathopoulos A, Koroneos A, et al. Increased levels of serum $S 100 B$ protein in critically ill patients without brain injury. Shock. $2006 \mathrm{Jul} ; 26(1): 20-4$

18 Mondal N, Bhat BV, Banupriya C, Koner BC. Oxidative stress in perinatal asphyxia in relation to outcome. Indian J Pediatr. 2010 May;77(5):515-7. 
19 Martin-Ancel A, Garcia-Alix A, Gaya F, Cabanas F, Burgueros M, Quero J. Multiple organ involvement in perinatal asphyxia. J Pediatr. 1995 Nov;127(5):78693.

20 Shah P, Riphagen S, Beyene J, Perlman M. Multiorgan dysfunction in infants with post-asphyxial hypoxic-ischaemic encephalopathy. Arch Dis Child Fetal Neonatal Ed. 2004 Mar;89(2):F152-F155.

21 Linnik MD,Zobrist RH, Hatfield MD. Evidence supporting a role for programmed cell death in focal cerebral ischemia in rats. Stroke. 1993 Dec;24(12):2002-8.

22 Zanelli SA, Numagami Y, McGowan JE, Mishra OP, Delivoria-Papadopoulos M. NMDA receptor-mediated calcium influx in cerebral cortical synaptosomes of the hypoxic guinea pig fetus. Neurochem Res. 1999 Mar;24(3):437-46.

23 Huang Z, Huang PL, Panahian N. Dalkara T, Fishman MC, Moskowitz MA. Effects of cerebral ischemia in mice deficient in neuronal nitric oxide synthase. Science. 1994 Sep 23;265(5180): 1883-5.

24 Nicholson DW, Thomberry NA. Caspases: killer proteases. Trends Biochem Sci. 1997 Aug;22(8):299-306.

25 Jajoo D, Kumar A, Shankar R, Bhargava V. Effect of birth asphyxia on serum calcium levels in neonates. Indian J Pediatr. 1995 Jul;62(4):455-9.

Basu P, Som S, Das H, Choudhuri N. Electrolyte status in birth asphyxia. Indian J Pediatr. 2010 Mar;77(3):259-62.

27 Aggarwal R, Upadhyay M, Deorari AK, Paul VK. Hypocalcemia in the newborn. Indian J Pediatr. 2001 Oct;68(10):973-5

28 Simovic AM, Knezevic J, Igrutinovic Z, Stojanovic N, Kocic S. [Cardiac troponin as biochemical marker of perinatal asphyxia and hypoxic myocardial injury]. Vojnosanit Pregl. 2009 Nov;66(11):881-6.

29 Lin LX, Mao QH, Zhang ZL, An CX, Kang XG. [Plasma levels of N-terminal pro-brain natriuretic peptide and glycogen phosphorylase isoenzyme BB in neonates with asphyxia complicated by myocardial injury]. Zhongguo Dang Dai Er Ke Za Zhi. 2010 Apr; 12(4):252-5.

30 Rajakumar PS, Vishnu BB, Sridhar MG, Balachander J, Konar BC, Narayanan P, et al. Electrocardiographic and echocardiographic changes in perinatal asphyxia. Indian J Pediatr. 2009 Mar;76(3):261-4

31 Rowe RD, Hoffman T. Transient myocardial ischemia of the newbom infant: a form of severe cardiorespiratory distress in full-term infants. J Pediatr. 1972 Aug;81(2):243-50

32 Lapointe A, Barrington KJ. Pulmonary hypertension and the asphyxiated newborn. J Pediatr. 2011 Feb;158(2 Suppl):el9-e24.

33 Leone TA, Finer NN. Shock: a common consequence of neonatal asphyxia. J Pediatr. 2011 Feb;158(2 Suppl):e9-12.

34 Tarcan A, Tiker F, Guvenir H, Gurakan B. Hepatic involvement in perinatal asphyxia. J Matern Fetal Neonatal Med. 2007 May;20(5):407-10.

35 Fischler B, Pettersson M, Hjern A, Nemeth A. Association between low Apgar score and neonatal cholestasis. Acta Paediatr. 2004 Mar;93(3):368-71.

36 Durkan AM, Alexander RT. Acute kidney injury post neonatal asphyxia. J Pediatr. 2011 Feb;158(2 Suppl):e29-e33

37 Gupta BD, Sharma P, Bagla J, Parakh M, Soni JP. Renal failure in asphyxiated neonates. Indian Pediatr. 2005 Sep:42(9):928-34

38 Chevalier RL, Campbell F, Brenbridge AN. Prognostic factors in neonatal acute renal failure. Pediatrics. 1984 Aug;74(2):265-72.

39 Kaur S, Jain S, Saha A, Chawla D, Parmar VR, Basu S, et al. Evaluation of glomerular and tubular renal function in neonates with birth asphyxia. Ann Trop Paediatr. 2011;31(2):129-34
40 Moake JL. Haemolytic-uraemic syndrome: basic science. Lancet. 1994 Feb 12;343(8894):393-7

41 Biran V, Fau S, Jamal T, Veinberg F, Renolleau S, Gold F, et al. Perinatal asphyxia may present with features of neonatal atypical hemolytic uremic syndrome. Pediatr Nephrol. 2007 Dec:22(12):2129-32.

42 Levi M, de JE, Meijers J. The diagnosis of disseminated intravascular coagulation. Blood Rev. 2002 Dec;16(4):217-23.

43 Salhab WA, Wyckoff MH, Laptook AR, Perlman JM. Initial hypoglycemia and neonatal brain injury in term infants with severe fetal acidemia. Pediatrics. 2004 Aug:114(2):361-6.

44 Basu P, Som S, Choudhuri N, Das H. Contribution of the blood glucose level in perinatal asphyxia. Eur J Pediatr. 2009 Jul;168(7):833-8.

45 Shankaran S, Laptook AR, Ehrenkranz RA, Tyson JE, McDonald SA, Donovan $\mathrm{EF}$, et al. Whole-body hypothermia for neonates with hypoxic-ischemic encephalopathy. N Engl J Med. 2005 Oct 13;353(15):1574-84.

46 Burden AD, Krafchik BR. Subcutaneous fat necrosis of the newbom: a review of 11 cases. Pediatr Dermatol. 1999 Sep;16(5):384-7.

47 van BF, Shadid M, Moison RM, Dorrepaal CA, Fontijn J, Monteiro L, et al. Effect of allopurinol on postasphyxial free radical formation, cerebral hemodynamics, and electrical brain activity. Pediatrics. 1998 Feb;101(2):185-93.

48 Levene MI, Gibson NA, Fenton AC, Papathoma E, Bamett D. The use of a calcium-channel blocker, nicardipine, for severely asphyxiated newbor infants. Dev Med Child Neurol. 1990 Jul;32(7):567-74.

49 Donn SM, Goldstein GW, Schork MA. Neonatal hypoxic-ischemic encephalopathy: current management practices. J Perinatol. 1988;8(1):49-52.

50 Hunt R, Osborn D. Dopamine for prevention of morbidity and mortality in term newborn infants with suspected perinatal asphyxia. Cochrane Database Syst Rev. 2002;(3):CD003484

51 Ichiba H, Tamai H, Negishi H, Ueda T, Kim TJ, Sumida Y, et al. Randomized controlled trial of magnesium sulfate infusion for severe birth asphyxia. Pediatr Int. 2002 Oct;44(5):505-9.

52 Davis PG, Tan A, O'Donnell CP, Schulze A. Resuscitation of newborn infants with $100 \%$ oxygen or air: a systematic review and meta-analysis. Lancet 2004 Oct 9;364(9442):1329-33.

53 Kattwinkel J, Perlman JM, Aziz K, Colby C, Fairchild K, Gallagher J, et al. Part 15: neonatal resuscitation: 2010 American Heart Association Guidelines for Cardiopulmonary Resuscitation and Emergency Cardiovascular Care. Circulation. 2010 Nov 2;122(18 Suppl 3):S909-S919.

54 Jones G, Steketee RW, Black RE, Bhutta ZA, Morris SS. How many child deaths can we prevent this year? Lancet. 2003 Jul 5;362(9377):65-71

55 Stuart A, Otterblad OP, Kallen K. Apgar scores at 5 minutes after birth in relation to school performance at 16 years of age. Obstet Gynecol. $2011 \mathrm{Aug} ; 118(2 \mathrm{Pt}$ 1):201-8.

56 WESTIN B, MILLER JA, Jr., NYBERG R, WEDENBERG E. Neonatal asphyxia pallida treated with hypothermia alone or with hypothermia and transfusion of oxygenated blood. Surgery. 1959 May;45(5):868-79

57 Shah PS. Hypothermia: a systematic review and meta-analysis of clinical trials. Semin Fetal Neonatal Med. 2010 Oct;15(5):238-46.

58 Nelson KB, Chang T, Ghadini A. Comment and reply on: Neonatal asphyxia and forensic medicine. J Matern Fetal Neonatal Med. 2011 Apr;24(4):652-3.

59 Locatelli A, Incerti M, Ghidini A, Greco M, Villa E, Paterlini G. Factors associated with umbilical artery acidemia in term infants with low Apgar scores at 5 min. Eur J Obstet Gynecol Reprod Biol. 2008 Aug;139(2):146-50. 